\title{
A photoacoustic imaging system employing a curved-phased ultrasonic array and parallel electronics
}

\section{Anastasios Maurudis, Fei Huang, Puyun Guo, Shikui Yan, Diego Castillo, et al.}

Anastasios Maurudis, Fei Huang, Puyun Guo, Shikui Yan, Diego Castillo, Lihong V. Wang, Quing Zhu, "A photoacoustic imaging system employing a curved-phased ultrasonic array and parallel electronics," Proc. SPIE 6086, Photons Plus Ultrasound: Imaging and Sensing 2006: The Seventh Conference on Biomedical Thermoacoustics, Optoacoustics, and Acoustooptics, 60861Q (6 March 2006); doi: 10.1117/12.648500

SPIE. Event: SPIE BiOS, 2006, San Jose, California, United States 


\title{
A photoacoustic imaging system employing a curved-phased ultrasonic array and parallel electronics
}

\author{
Anastasios Maurudis* ${ }^{\mathrm{a}}$, Fei Huang ${ }^{\mathrm{a}}$, Puyun Guo ${ }^{\mathrm{a}}$, Shikui Yan ${ }^{\mathrm{a}}$, Diego Castillo ${ }^{\mathrm{a}}$, Lihong V. Wang ${ }^{\mathrm{b}}$, \\ and Quing Zhu ${ }^{\mathrm{a}}$ \\ ${ }^{a}$ University of Connecticut, Biomedical Ultrasonic and Optical Imaging Group, Storrs, CT 06269 \\ b Texas A\&M University, Optical Imaging Laboratory, College Station, TX 77843
}

\begin{abstract}
Real-time photoacoustic imaging requires ultrasonic array receivers and parallel data acquisition systems for the simultaneous detection of weak photoacoustic signals. In this paper, we introduce a newly completed ultrasonic receiving array system and report preliminary results of our measured point spread function. The system employs a curved ultrasonic phased array consisting of 128-elements, which span a quarter of a complete circle. The center frequency of the array is $5 \mathrm{MHz}$ and the bandwidth is greater than $60 \%$. In order to maximize the signal-to-noise ratio for photoacoustic signal detection, we utilized special designs for the analog front-end electronics. First, the 128 transducer-element signals were routed out using a 50-Ohm impedance matching PCB board to sustain signal integrity. We also utilize 128 low-noise pre-amplifiers, connected directly to the ultrasonic transducer, to amplify the weak photoacoustic signals before they were multiplexed to a variable-gain multi-stage amplifier chain. All front-end circuits were placed close to the transducer array to minimize signal lose due to cables and therefore improve the signal-to-noise ratio. Sixteen analog-to-digital converters were used to sample signals at a rate of 40 mega-samples per second with a resolution of 10-bits per sample. This allows us to perform a complete electronic scan of all 128 elements using just eight laser pulses.
\end{abstract}

Keywords: Photoacoustics, optoacoustics, thermoacoustics, laser-generated ultrasound, biomedical imaging systems, parallel electronics.

\section{INTRODUCTION}

The photo-acoustic phenomenon was first reported by Alexander Graham Bell in 1880. However, only within the past decade has photo-acoustic imaging emerged as a promising biomedical imaging modality. Photo-acoustic imaging studies have been performed using tissue phantoms [1-4], in-vitro [5] and in-vivo [6-7]. These studies, among others, show that photo-acoustic imaging has the capability to image heterogeneous tissue structures with a sub-millimeter resolution at depths up to several centimeters [3].

The ultimate goal of our photoacoustic imaging system is to provide a means for the medical community to detect cancer tumors in an early stage. Generally speaking, the survival rate of a patient increases as the tumor size and/or tumor life decrease. Thus, early detection of said cancer tumors is critical. The spawning and survival of a cancer tumor is highly dependent upon angiogenesis. Angiogenesis is formally defined as the process of vascularization of a tissue involving the development of new capillary blood vessels. Thus, the angiogenesis process improves the initial oxygen and nutrient supply of the cancer tumors via these newly formed micro blood vessels. As pointed out in [8], angiogenesis is required for invasive tumor growth and metastasis and constitutes an important point in the control of cancer progression. Thus, the inhibition of angiogenesis is a desirable outcome of cancer therapy. A lack of blood supply will diminish the probability of tumor growth and metastasis. In order to inhibit the angiogenesis process you must first be able to detect the angiogenesis and then monitor the sustainability of the vessels under a given cancer therapeutic method. Specifically a photoacoustic imaging system is able to detect the blood, e.g. hemoglobin, in the vessels associated with a given tumor's angiogenesis and provide the needed information to a health care expert. This is possible since known optical wavelengths exist such that the optical absorption rate of oxygenated hemoglobin and de-oxygenated hemoglobin is much higher, up to 10 times, compared to the surrounding biological tissue for certain cancer tumors, such as breast

*AM@ENGR.UCONN.EDU; phone 1860486 1818; fax 1860486 2447; www.engr.uconn.edu/ece/labs/optlab/

Photons Plus Ultrasound: Imaging and Sensing 2006: The 7th Conference on Biomedical

Thermoacoustics, Optoacoustics, and Acousto-optics, edited by A. A. Oraevsky, et. al

Proc. of SPIE Vol. 6086, 60861Q (2006) • 1605-7422/06/\$15 • doi: 10.1117/12.648500 
cancer. Another point worth noting is that a malignant tumor will have more blood (i.e. hemoglobin) per unit volume, thus having a greater optical absorption rate compared to a benign tumor. The previous statement implies that a given photoacoustic imaging system has a higher average probability of detection for a malignant tumor compared to a benign tumor.

Our paper is organized as follows: in section 2, we give an introduction to the photoacoustic signal generation process from an imaging system designer's point of view. The photoacoustic imaging system is described in section 3 . The methods used to verify our photoacoustic imaging system are explained in section 4, with the results presented in section 5. Our summary and conclusion are given in section 6. At the time of publication, our laser source did not arrive from the manufacturer. Thus, our imaging system was characterized using a narrowband semi-spherical ultrasonic transducer source at anticipated photoacoustic signal levels.

\section{PHOTOACOUSTIC SIGNAL GENERATION}

The photoacoustic signal generation process involves the propagation of energy from an optical state to a thermal state to a mechanical state and finally an electrical state. A systematic view of the energy propagation/conversion is depicted in figure 1. In our photo-acoustic imaging system, a Q-switch pulsed Nd:YAG laser (Symphotic Tii Corp.) source will illuminate a biological tissue. The laser pulse duration is 10 nano-seconds with a pulse repetition frequency of $10 \mathrm{~Hz}$. The optical energy will be set below the maximum exposure for skin at the given wavelength. The biological tissue will absorb the photons at locations where absorbing molecules are present, such as in hemoglobin. When a photon is absorbed, an electron in the absorbing molecule is lifted to a higher energy level [9]. This is an all-or-nothing event; to be absorbed the photon must contain a quantity of energy that exactly matches the energy of the electronic transition. A molecule that has absorbed a photon is in an excited state, which is generally unstable. An electron lifted into a higher-energy orbital usually returns rapidly to its normal lower-energy orbital; the excited molecule decays to the stable ground state, giving up the absorbed quantum as light or heat or using it to do chemical work. In photo-acoustic imaging applications, the wavelength of the laser source is chosen such that the majority of the absorbed quantum is converted to heat (i.e. non-radiative energy) in an efficient manner. The conversion efficiency from optical to thermal can be $90 \%$ or greater [10]. The heat causes a local temperature rise in the absorbing tissue. The temperature rise causes the pressure to rise due to restricted expansion (i.e. volumetric changes), such as in blood vessels. The pressure wave will propagate to the body surface in a relatively non-dispersive pathway. Thus, a sensitive transducer element can detect the pressure wave and convert it to a voltage (i.e. an electronic signal). In other words, the optical energy is converted to thermal energy via photon absorption. The thermal energy is converted to mechanical energy (i.e. pressure waves) via thermo-elastic expansion. The mechanical energy is converted to electrical energy via the transducer. The mathematical equations and models used to describe the energy propagation of a photoacoustic signal have been reported extensively in the literature [10-14].

QUESTION OF THE DAY = I AM THE LIGHTINING! I AM THE HEAT! I AM THE WAVE! I AM THE CHARGE! WHAT AM I? ANSWER OF THE DAY = PHOTOACOUSTIC SIGNAL GENERATION

\begin{tabular}{|l|l|l|l|l|}
\hline $\begin{array}{l}\text { Laser Pulse } \\
\text { Source }\end{array}$ & $\begin{array}{l}\text { Heat via } \\
\text { Photon } \\
\text { Absorption } \\
\text { Optical } \\
\text { Energy }\end{array}$
\end{tabular}

Figure 1: Systematic block diagram of the photoacoustic signal generation process. 


\section{PHOTOACOUSTIC IMAGING SYSTEM DESCRIPTION}

Up to now, we have described how the input to our electronic system (i.e. the transducer output) is generated. Next, we will focus on the transducer array. The transducer array was custom manufactured by Imasonic to our own specifications[15]. The transducer array consists of 128-elements in a curved aperture such that they form a quarter of a circle (i.e. 90 degrees), with a radius of curvature equal to 25 millimeters. It is worth noting that our plans call for the use of a 512 elemental curved aperture that covers an entire circle with the same exact specifications as the present 128element except the number of elements involved. The transducer array specifications call for a center frequency of 5 $\mathrm{MHz}$ and a bandwidth greater than $60 \%$. The physical dimensions of the elements are given by an elemental elevation aperture of 10 millimeters and a lateral pitch of about a wavelength. The minimum nearest neighbor acoustic crosstalk is specified to be -45 decibels. Furthermore, each element shall focus the acoustic sensitivity field in the elevation direction but unfocused in the azimuth (i.e. lateral) direction. The elevation focal plane shall be at the mid-point of the elevation aperture with a focal depth of 19 millimeters. The combined focal points of all elements will form a quarter-circle with a radius of curvature of 6 millimeters. The elevation focusing is accomplished by a direct shaping of the active material (piezocomposite technology) and does not use any acoustic lens. A top-view photograph of the array is shown in figure 2 such that the array elements are at the top of the photograph.

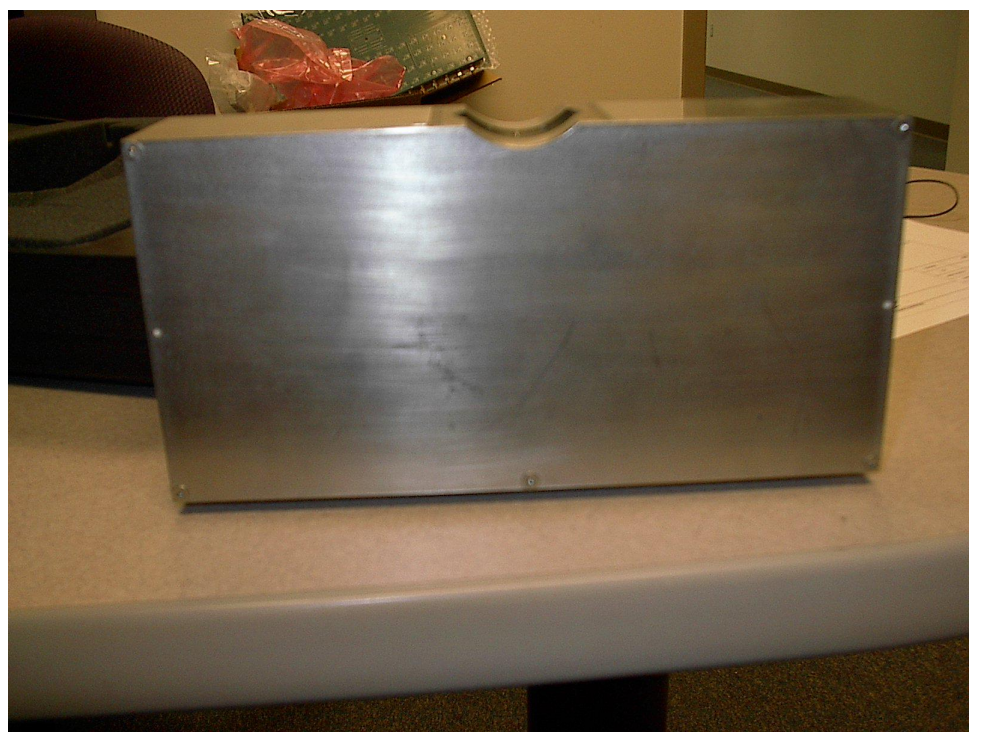

Figure 2: Photograph of the 128 elemental curved array.

Next we will give a brief description of the electronics associated with our imaging system. The reader is referred to [16] for a more detailed discussion. A system block diagram of our imaging setup is shown in figure 3 with an actual photograph shown in figure 4. Since the electronically-phased transducer array consists of 128 individual elements, our electronic system has 128 inputs (i.e. channels). As discussed in [16], an important design goal of the electronic system is to ensure that none of the 128 signals are severely distorted to cause any degradation in the quality of the electronic system output. More specifically, the purpose of the electronic system is to properly trigger data collection for digitizing and storing the output from each of the 128 transducer elements such that any alteration of the 128 signals due to the electronics is minimized. Due to time and budget constraints the data acquisition system (ADC) has only 16 individual analog-to-digital capture channels. Thus, we were forced to exploit a chose 1 out of 8 multiplexer (8:1) for each ADC input channel. The multiplexer allows us to select which 16 out of the 128 electronic system inputs will be captured for the present laser firing data capture session. Hence, we need a total of 8 firings to capture all 128 transducer elements.

For our imaging experiments, a personal computer will trigger the laser pulse to start illuminating the biological specimen at a pulse firing rate of 10 firings per second and a laser pulse width of 10 nano-seconds. The choice of a short pulse life is to ensure the pulse duration is less than the thermal diffusion time-constant associated with the biological specimen. For human biological tissue, a pulse duration of less than $1 \mu$-sec is assumed to avoid thermal diffusion $[1,13]$. Next, the laser source will trigger the data acquisition system (ADC) as each laser pulse is fired. In essence, the laser source is telling the data acquisition system when to start a data capture session. For us, the time-length of a single data capture session is configurable and limited by the amount of on-board memory. 
Once the data acquisition system has initialized a data capture session, it will trigger the backplane controller to start applying a user configurable time-gain control pattern. Of course, the time-gain control pattern/waveform should not contain any sharp transitions or discontinuities to ensure the voltage gain amplifier applies the gain correctly. That is, there is a time-lag for the amplifier to catch up with an instantaneous shift in the actual applied gain. Secondly, a true time-varying gain control signal is only necessary if the dynamic range of the low noise amplifier (i.e. LNA in figure 3) output over which the SNR remains satisfactory exceeds the dynamic range of the data acquisition system. That is, it is possible that for a given experiment the TGC signal is at a fixed gain (e.g. small volume of tissue with a small ultrasonic attenuation coefficient, coupled with a nearby transducer focal zone).

After the completion of a given data capture session, the data acquisition system triggers the backplane to switch the multiplexer address lines to the next set of 16 transducer elements for the subsequent data capture session. The backplane controller has direct access to the three address lines of all the multiplexers. The process is repeated until all 128 elements are captured. Upon completion, the data is moved to the PC via the PCI bus and stored in a file for postprocessing and image reconstruction.

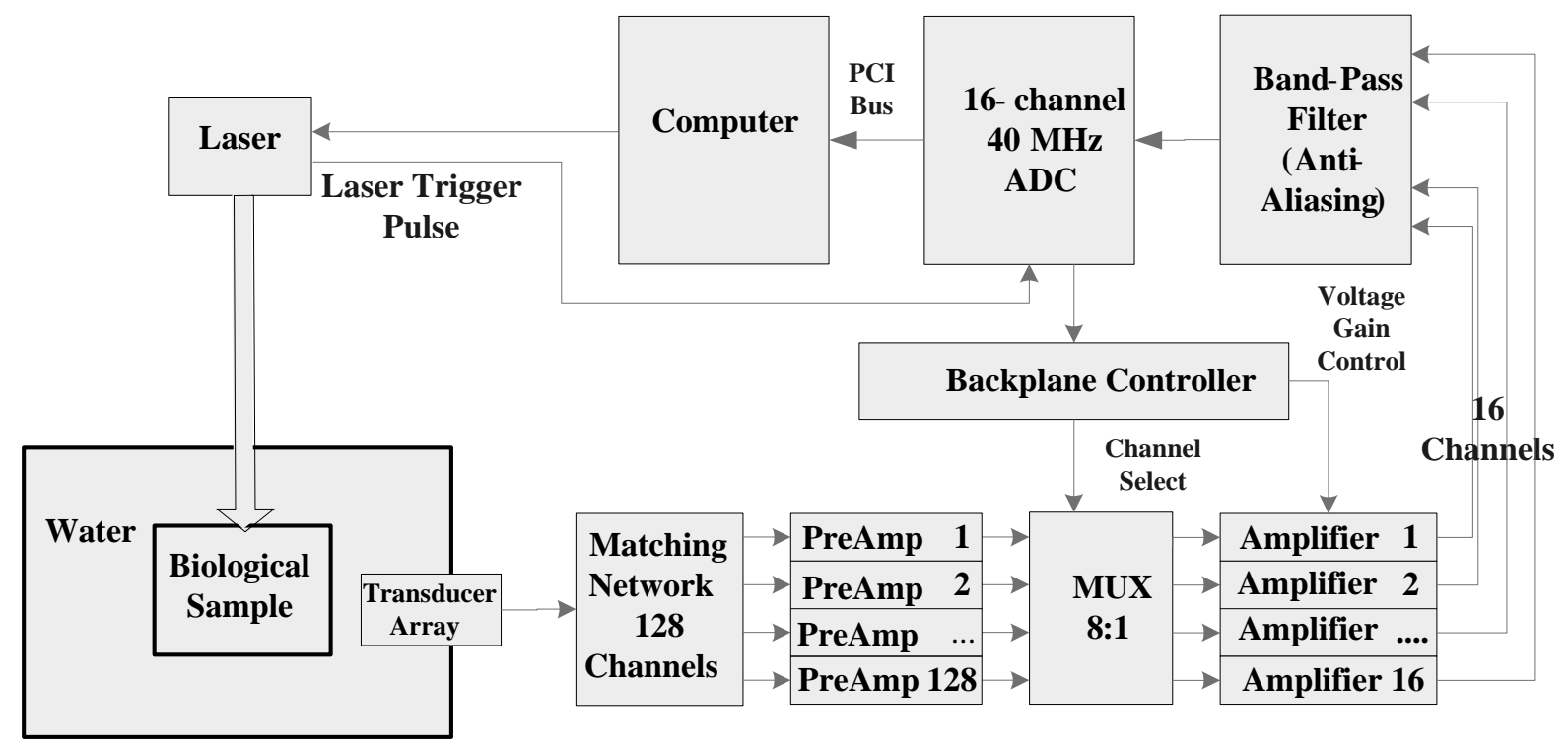

Figure 3: Block diagram of the photoacoustic imaging system.

\section{SYSTEM VERIFICATION METHODS}

The main sources of signal degradation caused by the electronic system are noise (thermal and quantization), crosstalk, amplitude distortion, and phase distortion [16-18]. The first priority was to verify the electronics used to support the photoacoustic imaging application. Once the individual electronic modules were verified under unit-testing methods, the complete system was integrated in well-chosen steps. The integrated system was verified by measuring the point spread function. For sake of brevity, not all verification methods we employed in our development are disclosed in this paper. However, a more detailed but yet limited discussion of said verification methods is given in [16].

The analog front end (AFE) consists of all the electronics in the pathway from the transducer output to the data acquisition system input. For the analog front end, we measured the magnitude response, signal-to-noise ratio (SNR), noise figure and the crosstalk over the bandwidth of interest (1 MHz to $10 \mathrm{MHz})$. These measurements were performed using a network analyzer (50-ohm sourced). The measurements were performed for each of the 128 channels over a range of gain levels, $G_{R x},=\{54,64,74,84,94\}$ in decibels.

The magnitude response was measured by supplying the AFE input channel selected by the multiplexer with the network analyzer signal output. The output of the AFE was fed to the network analyzer input terminal with all nonconnected nodes were terminated with $50 \Omega$. The magnitude response and the group delay are determined internally by the network analyzer. The magnitude response allows us to know the extent of the amplitude distortion introduced by the electronic pathway of the AFE. Under these test conditions, the parameterization of our system can incorporate the 


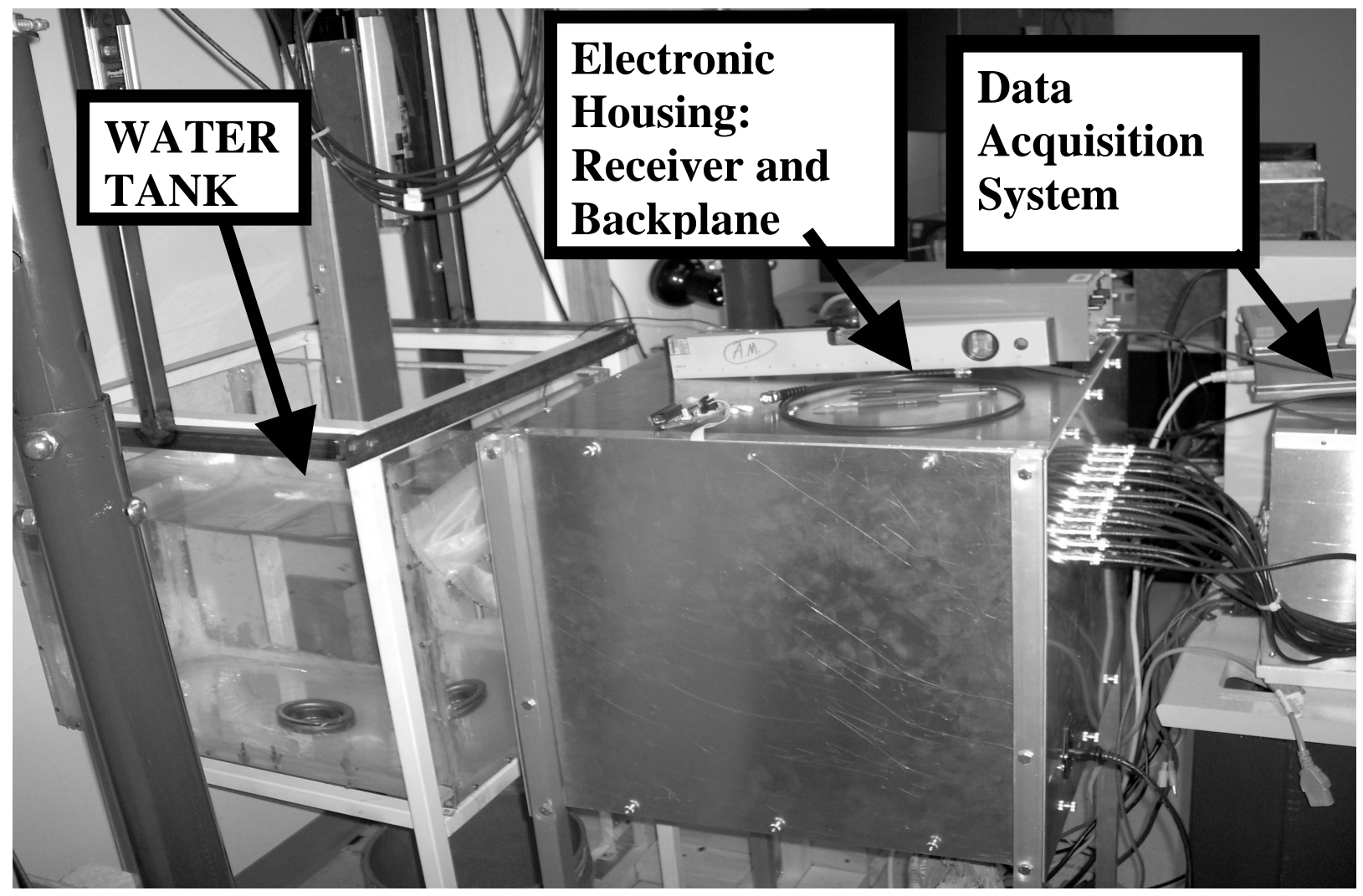

Figure 4: A photograph of our photoacoustic imaging system and experimental set-up.

complete S-parameter matrix (i.e. transmission and reflection coefficients) as a function of frequency [20].

The total SNR and the SNR density are derived from the signal spectral density and the noise spectral density. Specifically, the total SNR is the ratio of the integrated signal spectral density to the integrated noise spectral density over the bandwidth of interest. The total SNR is a single value (i.e. scalar) for a given test condition. On the other hand, the SNR density is the ratio of the signal spectral density to the noise spectral density on a per hertz basis. The SNR density is a vector of points sampled in the frequency domain. Thus, using the network analyzer we can measure the noise spectral density and the signal spectral density. Under the first set of conditions, the signal spectral density and noise spectral density were measured for a 50-ohm source impedance. The source impedance is seen looking out of the AFE input port. Of course, we know that for the actual photoacoustic imaging system the source impedance seen by the AFE input port is the transducer impedance which is not necessarily equal to 50-ohms.

Our next goal was to obtain the signal spectral density and noise spectral density at the AFE output based on the transducer source impedance. One way to accomplish this is to determine the Thevenin or Norton equivalent of the transducer element. The transducer array manufacturer (Imasonic) provided us the sensitivity (i.e. pressure-in to voltageout mapping) for a $50 \mathrm{ohm}$ load condition. Thus, we can determine the open circuit output voltage for a given input pressure field. The open circuit output voltage is the Thevenin equivalent voltage source. The Thevenin impedance and/or Norton admittance may be determined by measuring the reflection coefficient at the transducer array output port. Thus, one can easily generate a Norton or Thevenin circuit model for a given transducer element. As expected the circuit elements are not fixed values but a function of frequency. The other reason for knowing the transducer impedance is to determine the theoretical output noise spectral density of the AFE. The low noise amplifier at the first stage of the AFE is an active device whose output noise is directly dependent upon the impedance at its respective input port [16-19]. Finally, we determined the noise figure under the two conditions discussed, transducer sourced and 50-ohm sourced. The noise figure is simply the ratio of the input SNR divided by the output SNR. Thus, minimizing the system noise figure is desirable. 
Finally, the system integrated photoacoustic imaging system was verified by measuring the point spread function. As stated earlier, the laser was not available prior to publication, thus, we utilized a semispherical source. Moreover, the semispherical source is relatively narrowband compared to the bandwidth of the 128-elemental transducer array. Thus, the measured point spread function is more accurately the true point spread function convolved with the narrowband source. The ultrasonic source was placed in the focal zone of the middle two elements. The source location was controlled by our 3-D motor positioner system. The point spread function was determined by applying a non-apodization delay and sum beamforming algorithm. We know that other beamforming techniques exist which have the capability of improving the resolution [22].

\section{SYSTEM VERIFICATION RESULTS}

The expected magnitude responses for a given variable gain level setting are shown in figure 5. By "expected" we imply the expectation operator in the sense that we have averaged the measurement across all 128 channels. That is, the average response across 128 independent processes, thus ergodicity is not assumed. Clearly, the response is relatively flat within our bandwidth of interest. Thus, the corresponding amplitude distortion caused by our electronics is minimal.

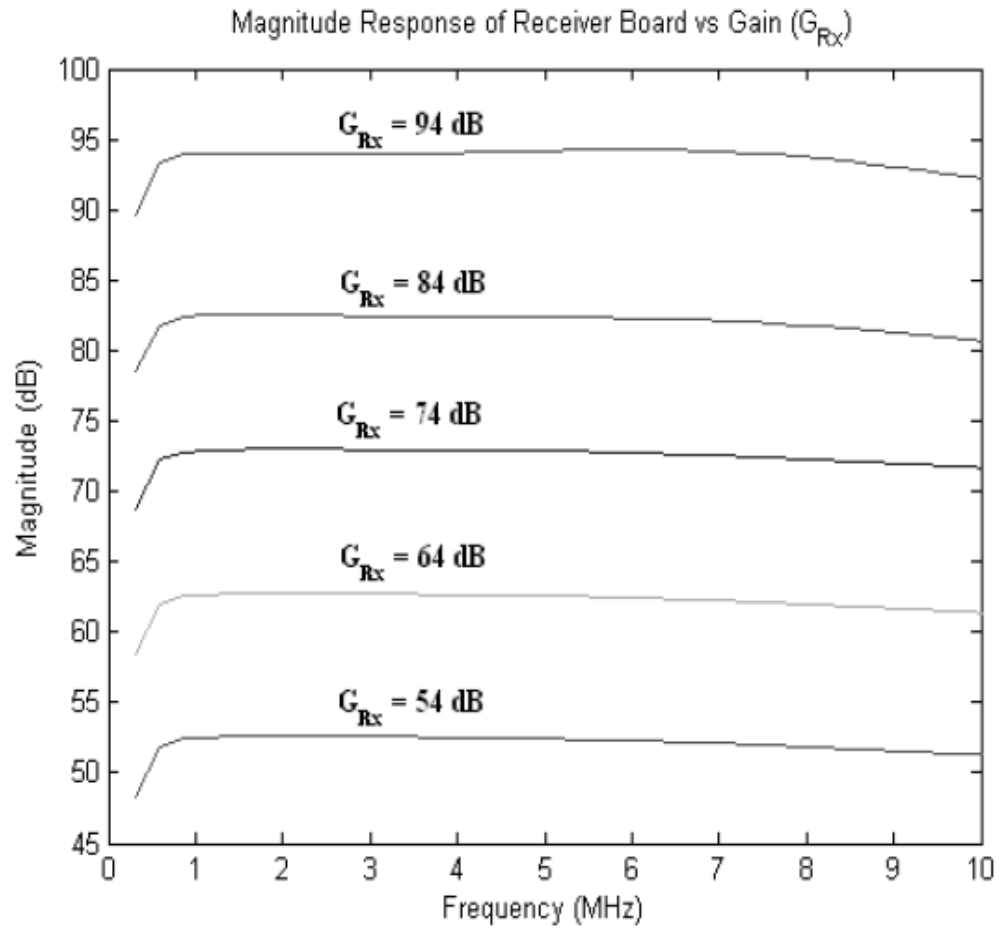

Figure 5: Average measured magnitude response of the electronic AFE pathway.

Next, we measured the reflection coefficient looking into the output port of each of the 128 transducer elements. From the reflection coefficient, we determined the Norton admittance. The Norton parallel conductance is shown in figure $6 \mathrm{a}$ and the Norton parallel capacitance is shown in figure 6b. Next, we determined the open circuited voltage for the "average" transducer element, based on data supplied by the transducer array manufacturer, for a broadband pressure wave at 40 Pascals. The voltage level appearing at the first stage low-noise amplifier input port can be determined using the common voltage divider approach. We can accomplish this since we know the transducer Thevenin voltage, transducer Norton admittance and the input impedance of low-noise amplifier. The plot of the open-circuit voltage and the voltage at the low-noise amplifier (LNA) input port is shown in figure 7a, with respective noise spectral densities shown in figure $7 \mathrm{~b}$. Next, the SNR density and the associated noise figure for the transducer source case are shown in figure 8. The noise figure for the 50-ohm sourced case was at a constant $7 \mathrm{~dB}$; not frequency dependent within our bandwidth of interest. The crosstalk plots are shown in figure 9. Since we do not expect the input levels to ever approach 

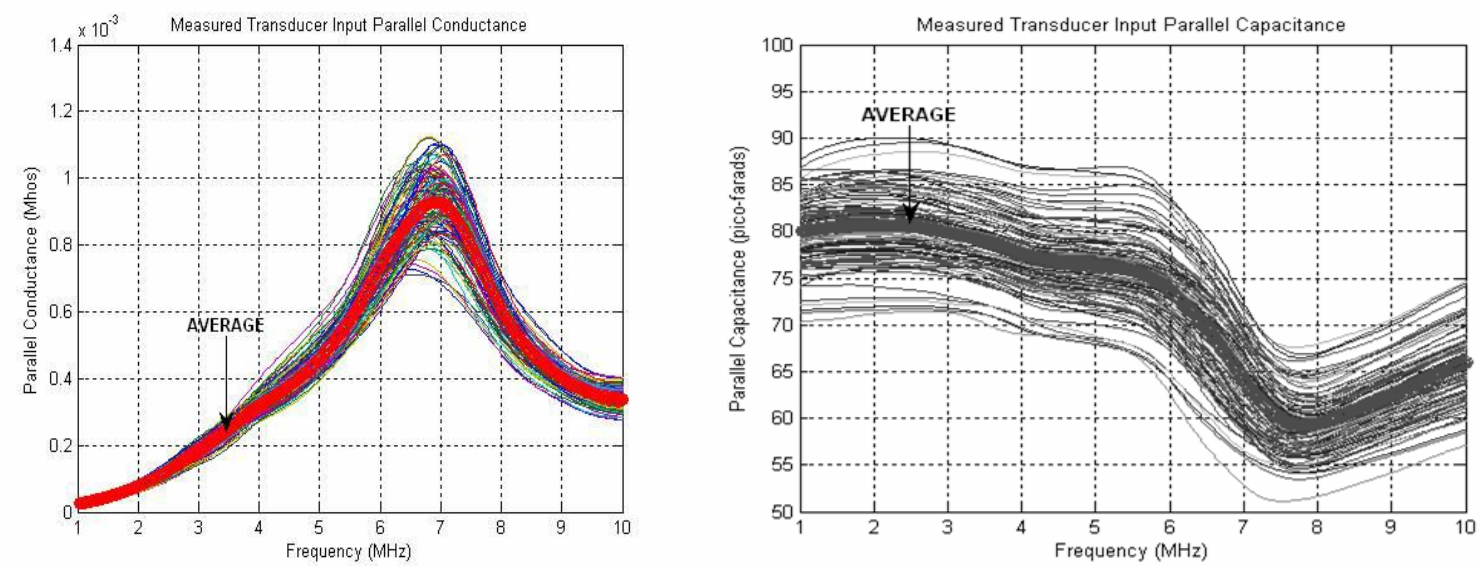

Figure 6a: Measured Norton parallel conductance, in Mhos, of the 128 transducer elements.

Figure 6b: Measured Norton parallel capacitance, in pico-farads, of the 128 transducer elements.
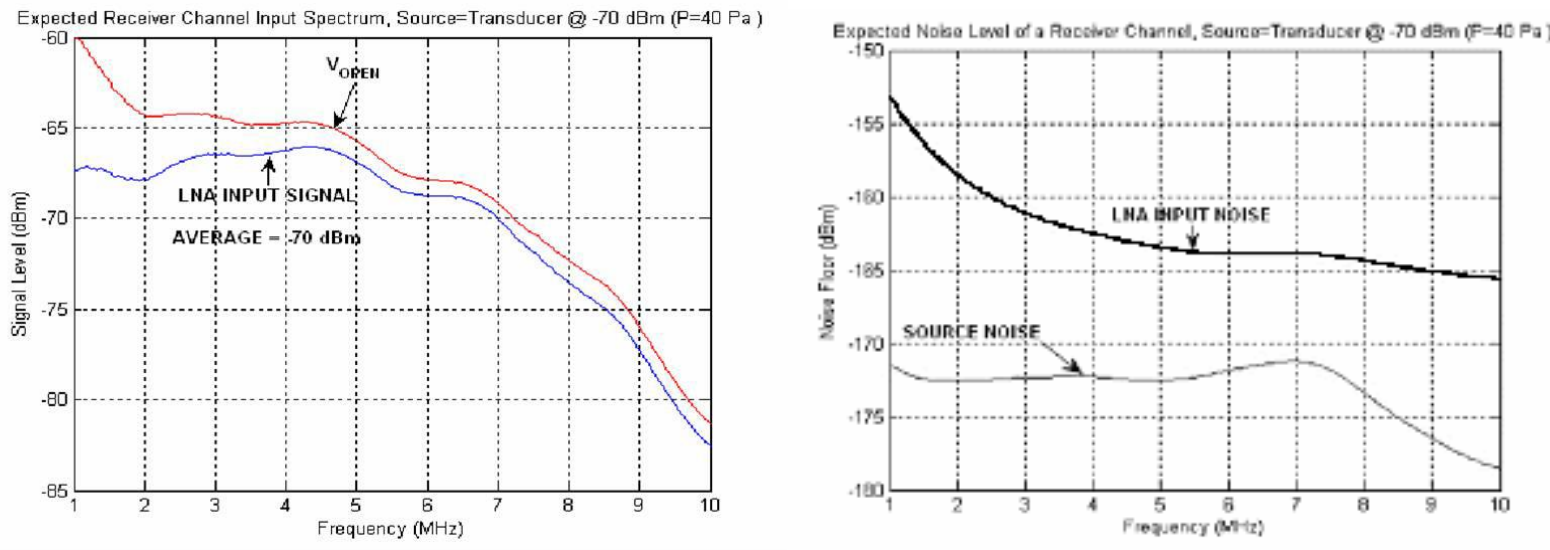

Figure 7a: Signal spectral density, open-circuited and amplifier input port.

Figure 7b: Noise spectral density: transducer sourced and LNA input noise (referred to input).
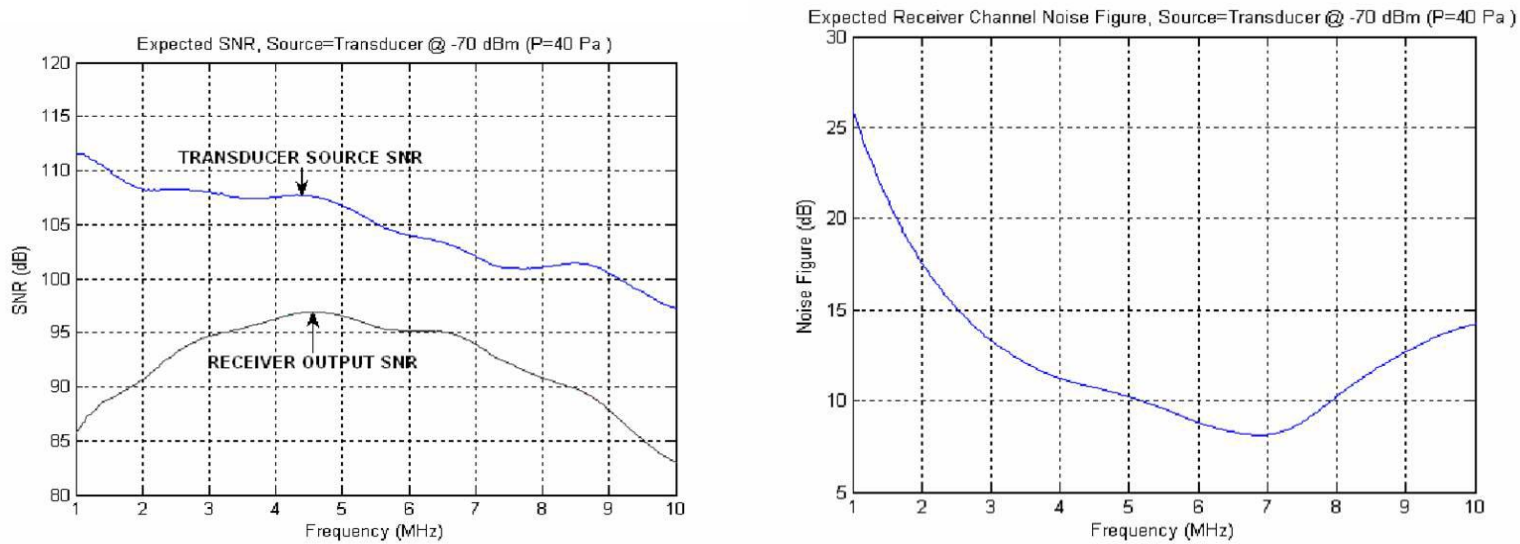

Figure 8a: SNR density at the transducer output port and the data acquisition input port.

Figure 8b: Noise figure for the transducer sourced case. 


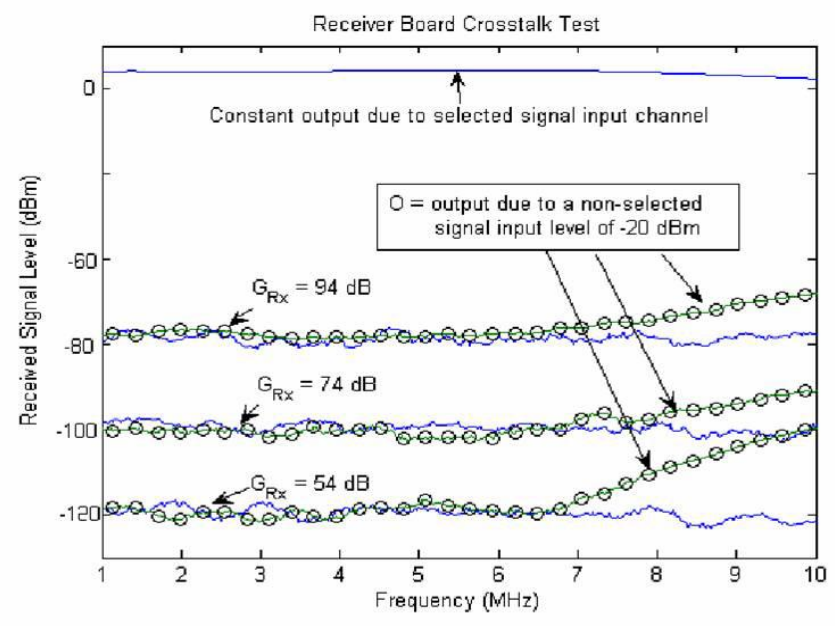

Figure 9: Crosstalk measurements for gain settings of $\{54,74,94\} \mathrm{dB}$.

$-20 \mathrm{dBm}$ for the gain levels tested, the crosstalk will always be below the noise floor.

Next, we show, in figure 10, the output frequency spectrum of the semi-spherical transducer source used to measure the point spread function. Clearly, the spectrum is narrowband relative to the bandwidth of interest (1-10 MHz). Thus, as expected the axial resolution is limited by the time-spreading of the impulse response of the narrowband source. The two-dimensional measured point spread function is shown in figure 11. The origin of our coordinate system was chosen at the semi-spherical ultrasonic source location. The lateral beam profile and axial beam profile are shown in figure 12 . The lateral beamwidth approaches 200 microns with the axial beamwidth around 2 millimeters.

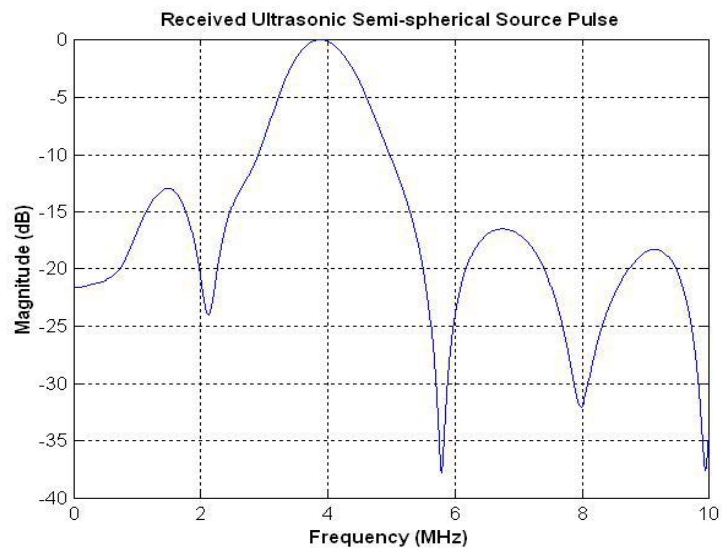

Figure 10: Semi-spherical ultrasonic source frequency spectrum. 


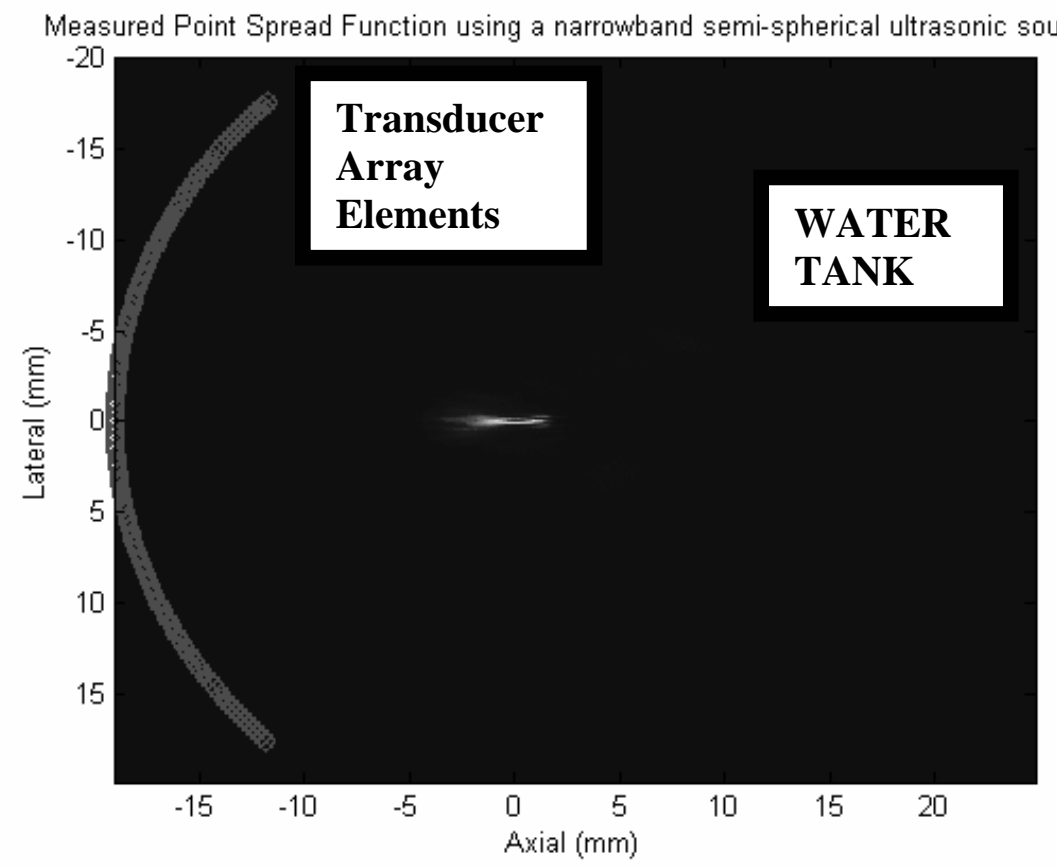

Figure 11: Measured point spread function using a narrowband semi-spherical ultrasonic source.
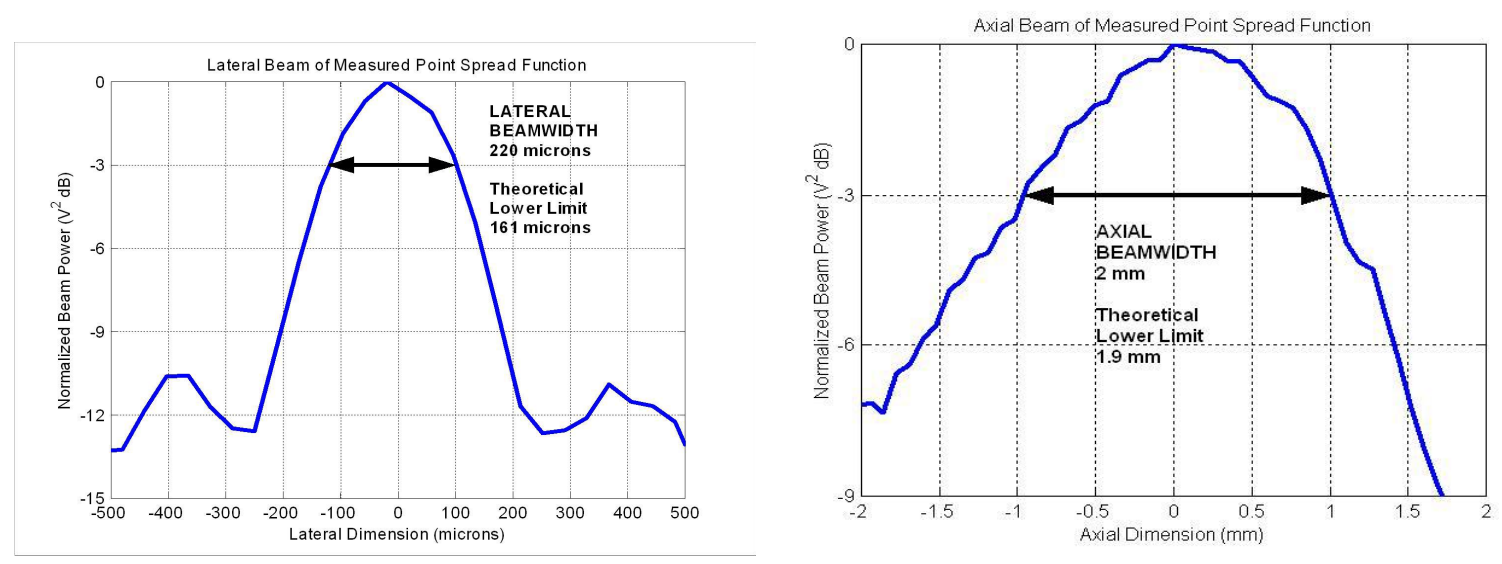

Figure 12: Lateral beam profile and axial beam profile of the measured point spread function.

\section{SUMMARY AND FUTURE WORK}

We have extensively verified the electronic system components, both as stand-alones and integrated as one imaging system. The electronics can support a high enough SNR, at the given weak photo-acoustic generated input levels, to provide a quality image. This will further the case for photoacoustic methods to be utilized as an imaging modality by healthcare specialists in the battle against cancer.

Next, we will use the laser and a point absorber with a known optical absorption rate to measure the point spread function within a 3-dimensional voxel. The location of the point absorber will be controlled by our 3-D motor control system. The motor control system is integrated within a NI LabView environment for ease of testing. Once the point spread function is measured we will move on to measuring tissue phantom to further characterize the quality and resolution of our imaging system. Finally, we will move onto in-vivo studies. 
Our goal is to expand this design in our 512-elemental ring array system. In that system, the transducer array forms a complete circle, hence the name ring array, instead of just a quarter of a circle as in this 128-elemental array system. The 512 elemental ring array system can be thought of as four 128-elemental array systems. When we designed the 128elemental array system, we had scalability in mind since we knew we would extend the 128 system to a complete 512 elemental circle. Our data acquisition system is designed to receive 64 channels (not just 16); the number required in the 512 elemental system using an 8:1 multiplexer approach (i.e. 512 divided by 8 is 64).

No matter how tough one may think it is doing innovative research, staying up late, getting stressed out, etc., it does not compare to battling cancer, going through chemotherapy, or radiation therapy (i.e. cancer sucks because cancer kills!).

\section{ACKNOWLEDGEMENTS}

The work was supported by the National Institute of Health under contract R01 NS46214. The authors wish to thank Mr. Pierre Dufilie for his consultation on our electronic design, Ramakrishna Parchuri and Jaime Villamarin for their aid in testing our imaging system and all our group members for their help.

\section{REFERENCES}

1. R.A. Kruger, P. Liu, Y.R. Fang and C.R. Appledorn, "Photoacoustic ultrasound (PAUS)-reconstruction tomography," Med. Phys., vol. 22, pp. 1605-1609. October 1995

2. X.D. Wang, Y. Xu, M.H. Xu, S. Yookoo, E.S. Fry and L.H.V. Wang, "Photoacoustic tomography of biological tissues with high cross-section resolution: Reconstruction and experiment," Med. Phys, vol. 29, pp. 2799-2805, December 2002.

3. S.A. Carp, A. Guerra, S.Q. Duque, and V. Venugopalan, "Optoacoustic imaging using interferometric measurement of surface displacement," App. Phy. Letters, vol. 85, pp.5772-5774, December 2004.

4. R.O. Esenaliev, A.A. Karabutov, and A.A. Oraevsky, "Sensitivity of laser opto-acoustic imaging in detection of small deeply embedded tumors," IEEE Journal Sel. Top. Quantum Elect. 5, Vol. 4, pp. 981-988, July-August 1999.

5. R.A. Kruger, W.L. Kiser, D. Reinecke, G.A. Kruger, and K.D. Miller, "Thermoacoustic molecular imaging of small animals," Mol. Imaging, vol. 2, pp. 113-123, April 2003.

6. X.D. Wang, Y.J. Pang, G. Ku, X.Y. Xie, G. Stoica and L.H.V. Wang, "Noninvasive laser-induced photoacoustic tomography for structural and functional in vivo imaging of the brain," Nat. Biotechnol., vol. 21, pp. 803-806, July 2003.

7. X.D. Wang, Y.J. Pang, G. Ku, G. Stoica, and L.H.V. Wang, "Three-dimensional laser-induced photoacoustic tomography of mouse brain with the skin and skull intact," Opt. Letters, vol. 28, pp. 1739-1741, October 2003.

8. J. Folkman, "Role of angiogenesis in tumor growth and metastasis," Seminar Oncol., vol. 29 (6 Suppl. 16):15-8, Dec. 2002.

9. D. Nelson and M. Cox, Lehninger Principles of Biochemistry, $4^{\text {th }}$ ed, New York: Freeman, 2005.

10. A. A. Oraevsky, "Optoacoustic systems for medical imaging: From principles to clinical applications," SPIE Photonics West Short Couse 768, Feb 2006.

11. G.J. Diebold, T. Sun, and M.I. Khan, "Photoacoustic waveforms generated by fluid bodies," Photoacoustic and Photothermal Phenomena III, Springer-Verlag, Berlin, 1992.

12. G. Ku, X. Wang, G. Stoica, and L.V. Wang, "Multiple-bandwidth photoacoustic tomography," Phy. Med. Biol., vol. 49, pp. 1329-1338, March 2004.

13. J. Rippoll and V. Ntziachristos, "Quantitative point source photoacoustic inversion formulas for scattering and absorbing media,” Phy. Review, vol. 71, pp. 0319121-0319129, March 2005.

14. Y. Wang, D. Xing, Y. Zeng and Q. Chen, "Photoacoustic imaging with deconvolution algorithm," Phy. Med. Biol., vol. 49, pp. 3117-3124, July 2004.

15. A. Maurudis and Q. Zhu, "Ring transducer array specification for 128 elements, quarter circle system," Internal publication, University of Connecticut, Storrs, CT, Dec. 2004.

16. A. Maurudis, F. Huang, D. Castillo, P. Guo, S. Yan and Q. Zhu, "A novel electronic architecture used to support biomedical photo-acoustic imaging," Proc. 2006 IEEE International Symposium on Circuits and Systems (ISCAS 2006), May 2006. 
17. R.B. Northrup, Analysis and Application of Analog Electronic Circuits to Biomedical Instrumentation, $1^{\text {st }}$ ed., New York: CRC Press, 2004.

18. P.J. Fish, Electronic Noise and Low Noise Design, $1^{\text {st }}$ ed., New York: McGraw-Hill, 1994.

19. C.G. Oakley, "Calculation of ultrasonic transducer signal-to-noise ratios using the KLM model," IEEE Trans. Ultra. Ferr. Freq. Cont., vol. 44, pp. 1018-1026, September 1997.

20. G. Gonzalez, Microwave Transistor Amplifiers: Analysis and Design, $2^{\text {nd }}$ ed., New Jersey, Prentice-Hall, 1997

21. J.G. Proakis and D.G. Manolakis, Digital Signal Processing Principles, Algorithms, and Applications, $2^{\text {nd }}$ ed., New York: Macmillan, 1992

22. Z. Wang, J. Li, and R. Wu, "Time-delay- and time-reversal-based robust capon beamformers for ultrasound imaging,” IEEE Trans. Med. Imaging, vol. 24, no. 10, pp. 1308-1322, October 2005. 